\title{
INCLUSÃO NOS ESTAdOS UNIDOS: FILOSOFIA, IMPLEMENTAÇÃO E CAPACITAÇÃO DE PROFESSORES ${ }^{1}$ INCLUSION IN THE UNITED STATES: PHILOSOPHY; IMPLEMENTATION, AND CAPACITATING TEACHERS
}

Patricia J. PETERSON²

\begin{abstract}
RESUMO: a inclusão de alunos com deficiência em classes da educação geral é uma exigência importante das Leis para Indivíduos com Deficiência de 1975 e 1997 e da emenda da Lei para Indivíduos com Deficiência de 2004. Desde a promulgação dessas leis, as escolas públicas dos Estados Unidos têm sido obrigadas a incluir alunos com deficiência ao máximo possível em classes da educação geral onde eles possam estudar com pares não deficientes ao invés de passarem a maior parte de seu dia em classes segregadas com outros alunos com deficiência. Este trabalho discute a filosofia dos programas de inclusão e apresenta detalhes sobre programas de inclusão em um Estado específico dos Estados Unidos, o Estado do Arizona, Finalmente, descreve a necessidade de capacitação de professores para a área de educação geral e especial. Essa sessão demonstra porque os programas melhorados de capacitação de professores são necessários para aumentar o sucesso da inclusão nos Estados Unidos.
\end{abstract}

PALAVRAS-CHAVE: inclusão; capacitação de professores; ensino colaborativo.

\begin{abstract}
: inclusion of students with disabilities in general education classes is an important provision of the Individuals with Disabilities Education Act of 1975, 1997, and the Individuals with Disabilities Improvement Act of 2004. Since the inception of this law, public schools in the United States have been mandated to include students with disabilities to the maximum extent possible in general education classes where they can study with typical peers rather than spending most of their day in segregated classes with other students with disabilities. This paper will discuss the philosophy of these inclusion programs. In addition, the paper will present details about inclusion programs in a specific state within the United States, the state of Arizona. Finally, the teacher training need in the area of general education and special education collaboration will be described. This section will demonstrate why improved teacher preparation programs are needed in order to increase the success of inclusion in the United States.
\end{abstract}

KEYWORDS: inclusion; teacher preparation; colaborative teaching.

\section{INTRODUÇÃO}

O Estado do Arizona é um grande Estado, quase totalmente rural sendo que as duas grandes áreas urbanas no Arizona são Fênix e Tucson. A comparação do percentual de alunos servidos nos programas de inclusão será feita entre o Estado do Arizona e do resto do país. O trabalho também fará conexões entre os programas universitários de preparação de professores com sucesso de inclusão

\footnotetext{
${ }^{1}$ Texto traduzido por Maria Amelia Almeida (PPGEEs/UFSCar).

2 Professora de Educação Especial, Departamento de Especialidades Educacionais, Universidade do Norte do Arizona. Flagstaff, AZ USA 86011. Patricia.peterson@nau.edu
} 
nas escolas e descreverá a necessidade de mais programas de capacitação que preparem ambos, o educador geral e o educador especial em como implementar programas de inclusão colaborativa para alunos com deficiência matriculados em escolas de ensino fundamental e médio.

\section{Filosofia dos Programas de Inclusão}

Nos últimos anos, a educação de crianças com deficiência em classes comuns da escola regular tem sido uma prática promissora relacionada à implementação da reforma escolar (AUDETTE; ALGOZZINE, 1992; MCLESKEY; HENRY; HODGES, 1999; WALDRON; MCLESKEY; PACCHIANO, 1999). De acordo com Salend (2005), a filosofia de inclusão de alunos com deficiência em classes da educação geral cria um sistema educacional unificado no qual os alunos são educados juntos, em classes de alta qualidade e de acordo com a idade nas escolas locais. Para que seja eficaz, a inclusão deve envolver colaboração entre educadores, outros profissionais, alunos, famílias e agências da comunidade. A inclusão congrega a crença de que a instrução para alunos com deficiência deve ser desenvolvida com base em suas habilidades e não em suas inabilidades (FRIEND; BURSUCK, 2002). A inclusão não é apenas baseada na integração física, mas também nos princípios sociais de integração e integração instrucional (WILSON, 1999). O conceito subjacente de inclusão é que o professor da educação geral tem a responsabilidade principal de educar alunos com deficiência. Entretanto, se há uma justificativa no Programa de Educação Individualizada (PEI) do aluno, o mesmo também deve receber atendimento em outros ambientes, como, por exemplo, sala de recursos. Isso deve ser visto como um serviço auxiliar para as salas de educação geral, onde deve ser o local principal de colocação desses alunos. No interior da sala de aula da educação geral, além do educador, pode haver também outros tipos de suportes que vão desde o educador até o professor especializado (MASTROPIERI; SCRUGGS, 2004).

A emenda da Lei Educacional para Indivíduos com Deficiência (IDEIA), de 2004, é uma lei federal que oferece um forte apoio para inclusão de alunos com deficiência nas escolas públicas dos Estados Unidos. De acordo com Turnbull, Turnbull, Shank, and Smith (2004), a lei é baseada em seis princípios fundamentais que foram considerados uma espécie de pedra angular das leis anteriores (IDEA 1975 e 1997). Esses seis princípios são:1) rejeição zero; 2) avaliação não discriminatória; 3) educação gratuita e apropriada (FAPE); 4) ambiente o menos restritivo possível (LRE); 5) emolumentos judiciais; e 6) participação aluno/família. Dentre esses seis princípios, o conceito de ambiente o menos restritivo possível é um dos pontos essenciais do movimento de inclusão. Ambiente o menos restritivo possível (LRE) exige que o aluno com deficiência tenha o direito de ser educado em um ambiente no qual possa ser bem sucedido, que é muito parecido com o ambiente no qual os alunos não deficientes são educados (ETSCHEIDT; BARTLETT, 1999). 
O Vigésimo Terceiro Relatório Anual para o Congresso sobre a implementação da Lei Educacional para Indivíduos com Deficiência do Departamento de Educação dos Estados Unidos (Departamento de Educação dos Estados Unidos, 2001) relata que $76 \%$ dos alunos com todos os tipos de deficiência devem ser atendidos $40 \%$ do tempo ou mais em salas da educação geral. Esse relatório também oferece informações específicas sobre o percentual de alunos em cada categoria de deficiência que recebem atendimento $40 \%$ ou mais tempo na classe de Educação Geral: Dificuldades Específicas de Aprendizagem 83.5\%; Problemas de Fala ou Linguagem 95.1 \%; Retardo Mental 43\%; Distúrbios Emocionais 48.5 \%; Deficiências Múltiplas 27\%; Deficiência Auditiva 58.3\%; Impedimentos Ortopédicos 66.1\%; Outros Impedimentos de Saúde 77.5\%; Impedimentos Visuais 68.9\%; Autismo $33.5 \%$; Surdo-Cegueira 23.4\%; e Traumatismos Cerebrais $57.5 \%$.

\section{InClusão no Estado do Arizona}

Enquanto é importante olhar para os dados de todo o país relacionados aos programas de inclusão, também é interessante olhar como um Estado, de forma individual, oferece serviços para alunos com deficiência. O Estado do Arizona está localizado na região sudoeste dos Estados Unidos e faz fronteira ao sul com o México e a Oeste com o Estado da Califórnia. Os alunos com deficiência no Estado do Arizona provêm de ambientes culturais e lingüísticos diversos incluindo populações Hispânicas e Indígenas. O Departamento de Educação do Estado do Arizona dispõe de um relatório no qual consta que 104.524 alunos com deficiência foram atendidos em 2003. (Arizona Department of Education, 2003). Esse mesmo relatório também mostra dados sobre os tipos de serviços oferecidos a alunos com deficiência no Estado que podem ser comparados com dados nacionais, coletados pelo Departamento de Educação dos Estados Unidos (2001) sobre a porcentagem de alunos entre 6 a 21 anos matriculados em programas educacionais diferentes durante os anos escolares de 1998 - 1999. A comparação da porcentagem de alunos com deficiência em serviços específicos, tais como: A) Menos que $21 \%$ de seu tempo fora da sala regular - Arizona $47 \%$. Estados Unidos 48\%; B) 21 - 60\% de seu tempo

fora da classe regular - Arizona 32\%, Estados Unidos 28\%; C) Maior que $60 \%$ de seu tempo fora da sala regular - Arizona 18\%, Estados Unidos 20\%; D) Todo o tempo em instalação separada - escola dia - Arizona 2.6\%, Estados Unidos 3\%; E) Todo o tempo em instalação residencial - Arizona 1\%, Estados Unidos 5\%; e F) Todo o tempo em programa residencial/hospitalar - Arizona 3\%, Estados Unidos $5 \%$. Esses dados mostram que os serviços instrucionais para alunos com deficiência são oferecidos de maneira similar às porcentagens de alunos servidos, nacionalmente, em cada tipo de serviço nos Estados Unidos. 


\section{Educação Geral, Educação Especial, Inclusão e Necessidade de Capacitação de Professores em Ensino Colaborativo}

Enquanto a Lei Americana IDEA de 1997 foi delineada para servir, efetivamente, todos alunos com deficiência, ainda existe um número reduzido de professores qualificados para a educação especial em escolas nos Estados Unidos (Departamento de Educação dos Estados Unidos, 2002). Professores de educação especial totalmente certificados e altamente qualificados são imprescindíveis para a efetiva implementação de programas de inclusão para alunos com deficiência. De acordo com Brenner (1998), na última década ocorreu um número bem reduzido de professores totalmente certificados para a educação especial. Dois fatores significativos poderiam ter influenciado esse número reduzido de professores para a educação especial: 1) a população crescente de alunos da pré-escola e crianças em idade escolar que necessitam serviços de Educação Especial (Departamento de Educação Especial dos Estados Unidos, 2002); 2) o alto índice de professores da educação especial (FUCHS; FUCHS, 1995; ANDERSON; BAKER, 1999). Para complicar o assunto acerca de capacitação de professores, é conhecido que professores da educação geral são freqüentemente os primeiros que educam crianças com deficiências leves em suas salas de aula, que identificam as necessidades especiais desses alunos, que iniciam os procedimentos de encaminhamento, que participam do desenvolvimento do Programa de Educação Individualizado (PEI) e são os responsáveis pela implementação de programa educacional apropriado na classe de educação geral (HARDMAN; DREW; EGAN, 1999). Entretanto, a pesquisa mostra que os professores da educação geral acham que são preparados de forma inadequada para ensinarem, de maneira efetiva, crianças com deficiência inseridas nas salas de educação regular (TIEGERMAN-FARBER; RADZIEWICZ, 1998). Professores da educação geral indicam que a capacitação que receberam na universidade (disciplinas cursadas e experiência prática) não lhes forneceu conhecimento suficiente sobre métodos instrucionais e estratégias de manejo comportamental necessários para trabalhar, de forma segura, com alunos com uma variedade de deficiências (COOK; FRIEND , 1997; GABLE; HENDRICKSON, 1993; PETERSON; BELOIN, 1998; VOLK; STAHLMAN, 1994). Adicionalmente, a ênfase na inclusão de alunos com deficiência nas classes da educação geral, de acordo com a legislação de 1997 (IDEA, 1997), indica que os professores da educação geral têm maior necessidade de receber capacitação em serviço sobre métodos e técnicas colaborativas sobre como trabalhar com alunos com deficiência. Infelizmente, nas escolas americanas, os professores da educação geral indicam a falta de conhecimento sobre individualização de instruções para alunos com deficiência. Por um outro lado, professores da educação especial relatam a falta de conhecimento sobre o currículo da educação geral (LIPSKY; GARTNER, 1998).

Para que se possa atender alunos com deficiência de forma mais eficaz nas escolas americanas, todos os educadores americanos precisariam mudar sua mentalidade e pensar a educação especial como um serviço e não um lugar ou um rótulo. Serviços apropriados podem ser oferecidos em uma variedade de ambientes 
para uma variedade de indivíduos que tenham sido capacitados em métodos e técnicas para trabalhar com alunos com necessidades diversas (LIPSKY; GARTNER, 1998).

Para que se implemente efetivamente a inclusão, é necessário que haja uma completa reestruturação nas faculdades de educação das universidades americanas, da mesma forma que nas escolas de ensino fundamental e médio (BLANTON; GRIFFIN; WINN; PUGACH, 1997; PUGACH; SEIDL, 1998). As universidades, devem oferecer liderança visionária e assumir responsabilidade pelos resultados da inclusão (LIPSKY; GARTNER, 1998). Capacitação sobre as estratégias chaves para a inclusão deve ser oferecida para ambos, professores da educação geral e especial, da mesma forma para pais e alunos (CULATTA; TOMPKINS,1999).

Para realmente melhorar os resultados nos programas de inclusão para alunos com deficiência nas escolas, essa capacitação de professores de ser oferecida, não apenas nos programas de formação de professores nas universidades, mas também nas escolas locais para professores da educação geral (ARMSTRONG, 1996; SWANSON, 1999).

A literatura é clara sobre a necessidade imprescindível de capacitação sobre ensino colaborativo e inclusão (AUDETTE; ALGOZZINE, 1992; BENNER, 1998; BLANTON et al., 1997; CULATTA; TOMPKINS, 1999; HARDMAN et al, 1999; LIPSKY; GARTNER, 1998; SALEND, 2005; TIEGERMAN-FARBER; RADZIEWICZ, 1998). Na pressa de encontrar uma solução, erros sobre o conceito de inclusão ocorreram no passado. Esses erros estão relacionados à preparação inadequada dos participantes da inclusão, suporte insuficiente para os professores da educação geral, e comunicação inadequada entre os membros da equipe. (KAUFFMAN; HALLAHAN, 1994; COOK; FRIEND, 1993; FRIEND; COOK, 1997; LEWIS; DOORLAG, 2003).

Outra dificuldade para se promover inclusão e ensino colaborativo entre futuros professores é que os programas tradicionais de preparação de professores nas universidades preparavam os professores da educação geral de forma separada dos professores da educação especial com a desculpa de departamentos, classes, currículos e estágios separados. Se o objetivo nos Estados Unidos é o de reorganizar os programas de capacitação para facilitar colaboração entre educadores especiais e da educação geral que atendem alunos com deficiência em ambientes inclusivos, então os professores universitários têm que trabalhar técnicas de ensino colaborativo e inclusão em seus projetos e cursos (disciplinas) (BENNER, 1998). Todos os professores devem ter uma formação sólida sobre práticas bem sucedidas de inclusão em salas da educação geral. Essa formação inclui a crença em acesso eqüitativo para todos os alunos em termos de tratamento, oportunidade, e recursos (MONTECEL, 2000; SALEND, 2005). Além do mais, ambos professores da educação geral e especial precisam adquirir conhecimento sobre pesquisa e práticas eficazes necessárias para ensinar todos alunos com deficiência (por exemplo, ensino, 
precisão, manejo de sala de aula, transição da escola para o trabalho) (DESHLER, 2001; MERCER; MERCER, 2005; HEWARD, 2003; OVERTON, 2003).

\section{CONCLUSÃO}

Para que seja eficaz, a inclusão tem que envolver a colaboração entre professores especializados, professores da educação geral, administradores escolares, outros profissionais, alunos, famílias, agências da comunidade. A inclusão é baseada no constructo que instrução para alunos com deficiência deve ser desenvolvida em torno de suas habilidades e não de suas deficiências (FRIEND; BURSUCK, 2002). Inclusão não é apenas baseada em integração física, mas também em princípios de integração social e integração instrucional (WILSON, 1999).

O exemplo dos dados do Estado do Arizona (2003), um Estado que inclui uma população alta de crianças de ambientes cultural e lingüístico diferente vivendo em ambas, áreas urbanas e grandes áreas rurais, mostra que a implementação de programas específicos de inclusão podem ser comparados com a porcentagem de alunos com deficiência incluídos, nacionalmente, na educação geral.

Em relação à preparação de professores, os programas de inclusão não podem ser bem sucedidos em escolas públicas se ambos, professores de educação geral e especial não forem capacitados para implementar programas de ensino colaborativo e inclusivo na educação geral para alunos com deficiência. Para que esse objetivo seja atingido, os professores universitários devem modelar técnicas inclusivas e colaborativas em seus projetos e cursos (Benner, 1998). Todos os professores necessitam conhecer pesquisa e práticas eficazes para ensinar todos alunos com deficiência. Por meio dos achados deste trabalho, educadores de outros países que ainda não iniciaram programas de inclusão em suas escolas, talvez possam progredir olhando para as experiências, sucessos e desafios contínuos encontrados por professores de educação geral e especial que estão implementando programas de inclusão nos Estados Unidos.

\section{REFERÊNCIAS}

ANDERSON, P. L.; BAKER, B. K. A case-based curriculum approach to special education teacher preparation. Teacher Education and Special Education, v. 22, n. 3, p.188-192, 1999.

ARIZONA DEPARTMENT OF EDUCATION. Report on service types for students with disabilities. Phoenix, AZ: Author. 2003.

ARMSTRONG, C. E. The education of students with mental retardation in the United States. (ERIC Document Reproduction Service ED 395447). 1996.

AUDETTE, B. ; ALGOZZINE, B. Free and appropriate education for all students: total quality and the transformation of American Public Education. Remedial and Special Education, v. 13, n.6, p. 8-18, 1992. 
BENNER, S. Special education issues within the context of American Society. Belmont, CA: Wadsworth. 1998.

BLANTON. L.; GRIFFIN, C.; WINN, J.; PUGACH, M. Teacher education in transition: Collaborative programs to prepare general and special educators. Denver: Love, 1997.

COOK, L.; FRIEND, M. Educational leadership for teacher collaboration. In: BILLINGSLEY (Ed.) Program leadership for serving students with disabilities. Richmond, VA: Virginia Department of Education. 1993, p 421-444.

CULATTA, R. ; TOMPKIN, J. Fundamentals of special education: What every teacher needs to know. Columbus, OH: Merrill, 1999.

DESHLER, D. Instructional strategies for closing the performance gap. Paper presented November 3, 2001 Arizona CEC, Phoenix, AZ.

ETSCHEIDT, S.K.; BARTLETT, L. The IDEA amendment: A four-step approach to determining supplementary aids and services. Exceptional Children, v. 65, p. 163-174, 1999.

FRIEND, M.; BURSUCK, W. Including students with special needs: a practical guide for classroom teachers 3.ed. Boston, MA: Allyn and Bacon, 2002.

FRIEND, M.; COOK, L. Student centered teams in schools: still in search of an identity. Journal of Educational and Psychological Consultation, v.8, p. 3-20, 1997.

FUCHS, D.; FUCHS, L.S. What's "special" about special education? Phi Delta Kappan, v.76, p. 522-530, 1995.

GABLE, R. A. ; HENDRICKSON, J. M. Cooperative planning for regular classroom inclusion of students with disabilities. Preventing School Failure, v. 37, n. 4, p. 16-20, 1993.

HARDMAN, M., DREW, C. ; EGAN, M.W. Human exceptionality: Society, school, and family 6.ed. Boston: Allyn; Bacon, 1999.

HEWARD, W.L. Exceptional children: an introduction to special education. 7. ed. Upper Saddle River, NJ: Merrill/Prentice Hall, 2003.

KAUFFMAN, J. M.; HALLAHAN, D. P. Preface. In: KAUFFMAN, J. M.; Hallahan, D. P. (Eds.). The illusion of full inclusion, Austin, TX: PRO ED, 1994, p. 9-11.

LEWIS, R.; DOORLAG, D. Teaching special education students in general education classrooms. 6. ed. Upper Saddle River, NJ: Merrill/Prentice Hall, 2003.

LIPSKY, D.; GARTNER, A. Taking inclusion into the future. Educational Leadership, v. 56, n. 2, p. 78-81, 1998

MASTROPIERI, M.; SCRUGGS, T. The inclusive classroom: Strategies for effective instruction. 2. ed. Upper Saddle River, NJ: Merrill/Prentice Hall, 2004.

MCLEKSEY, J.; HENRY, D.; HODGES, D. Inclusion: What progress is being made across disability categories? Teaching Exceptional Children, v. 31, n. 3, p. 60-64, 1999.

MERCER, C. D.; MERCER, A. R. Teaching students with learning problems. 7. ed. Upper Saddle River, NJ: Merrill/Prentice Hall, 2005.

MONTECEL, M. Musical chairs and unkept promises. IDRA Newsletter, v. 27, p. 1-13, jan. 2000. 
OVERTON, T. Assessment in special education: an applied approach. 3. ed. Upper Saddle River, NJ: Merrill/Prentice Hall, 2003.

PETERSON, M.; BELOIN, K. S. Teaching the inclusive teacher: restructuring the mainstreaming course in teacher education. Teacher Education and Special Education, v. 21, n.4, p. 306-318, 1998.

PUGACH, M. C.; SEIDL, B. L. Responsible linkages between diversity and disability: a challenge for special education. Teacher Education and Special Education, v. 21, n. 4, p. 319333, 1998.

SALEND, S. Creating inclusive classrooms: effective and reflective practices 5. ed. Upper Saddle River, NJ: Merrill/Prentice Hall, 2005.

SWANSON, H.L. Instructional components that predict treatment outcomes for students with learning disabilities: support for a combined strategy and direct instruction model. Learning Disabilities Research and Practice, v. 14, n. 3, p. 129-140, 1999.

TIEGERMAN-FARBER, E.; RADZIEWICZ, C. Collaborative decision making: the pathway to inclusion, Columbus, OH: Merrill, 1998.

TURNBULL, R.; TURNBULL, A.; SHANK, M.; SMITH, S. Exceptional lives: special education in today's schools 4. ed. Upper Saddle River, NJ: Merrill/Prentice Hall, 2004.

UNITED STATES DEPARTMENT OF EDUCATION. Twenty-third annual report to Congress on the implementation of the Individuals with Disabilities Act. Washington, D.C.: Author. 2001.

UNITED STATES DEPARTMENT OF EDUCATION. Designated teacher shortage areas. Available http: http:// www.ed.gov/offices/OPE/Students/repayment/teachers/tsa.html acesso em 2002.

VOLK, D.; STAHLMAN, J. I think everybody is afraid of the unknown: early childhood teachers prepare for mainstreaming. Day Care and Early Education, v. 21, n. 3, p. 13-17, 1994.

WALDRON, N. L.; MCLESKEY, J.; PACCHIANO, D. Giving teachers a voice: teacher's perspectives regarding elementary inclusive school programs. Teacher Education and Special Education, v. 22, n. 3, p. 141-153, 1999.

WILSON, B. A. Inclusion: empirical guidelines and unanswered questions. Education and Training in Mental Retardation and Developmental Disabilities, v. 34, p. 119-133, 1999.

Recebido em 27/04/2006

Aceito em 30/04/2006 